\title{
On 4-manifolds which admit geometric decompositions
}

\author{
By Jonathan A. HiLlmaN
}

(Received Apr. 22, 1996)

\section{Introduction.}

An $n$-manifold $X$ is geometric in the sense of Thurston if its universal covering space $\tilde{X}$ admits a complete homogeneous Riemannian metric, $\pi_{1}(X)$ acts isometrically on $\tilde{X}$ and $X=\pi_{1}(X) \backslash \tilde{X}$ has finite volume. Every closed 1- or 2-manifold is geometric. Much current research on 3-manifolds is guided by Thurston's Geometrization Conjecture, that every closed irreducible 3-manifold admits a finite decomposition into geometric pieces [Th82]. There are 19 maximal 4-dimensional geometries; one of these is in fact an infinite family of closely related geometries and one is not realized by any closed 4-manifold [F]. Our first result (in \$1) shall illustrate the limitations of geometry in higher dimensions by showing that a closed 4-manifold which admits a finite decomposition into geometric pieces is usually either geometric or aspherical. The geometric viewpoint is nevertheless of considerable interest in connection with complex surfaces [Ue90,91, W185,86]. We show also that except for the geometries $S^{2} \times H^{2}$, $H^{2} \times H^{2}, H^{2} \times E^{2}$ and perhaps $\tilde{S L} \times E^{1}$ no closed geometric manifold has a proper geometric decomposition. In the rest of the paper we investigate connections between geometric decompositions and fibrations. In $\$ 2$ we characterize algebraically the homotopy types of (orientable) Seifert fibred 4-manifolds. This class of 4-manifolds includes all but three of the 4-dimensional infrasolvmanifolds with one of the geometries $E^{4}, N_{i l}^{4}, N^{3} l^{3} \times E^{1}$ or $S_{o l}^{3} \times E^{1}$ and all the manifolds with geometry $S^{3} \times E^{1}, S^{2} \times E^{2}$, $H^{2} \times E^{2}$ or $\tilde{S L} \times E^{1}$, but also has infinitely many nongeometric members. We give examples of such manifolds which have geometric decompositions but are not geometric, and also examples which do not have geometric decompositions. In $\$ 3$ we give criteria for a closed 4-manifold to be (homotopy equivalent to one which is) finitely covered by a cartesian product of closed hyperbolic surfaces. The final section determines when a 4-manifold which fibres over an aspherical closed surface with fibre a hyperbolic surface admits a geometry or a proper geometric decomposition. (Our result is incomplete in that we give only a necessary condition for such a manifold to admit one of the geometries $H^{4}$ or $H^{2}(C)$. There are no known examples of such bundle spaces).

AMS Subject Classification (1991): Primary 57N13. Secondary 53C30

Key words and phrases: 4-manifold. geometry. Seifert fibration. surface bundle. 


\section{§1. Geometric decompositions.}

We shall say that an $n$-manifold $M$ is geometric if it is geometric in the sense of Thurston, and more generally that it admits a geometric decomposition if it may be split along a finite collection of disjoint 2-sided hypersurfaces $S$ such that each component of $M-U S$ is geometric. We shall call the hypersurfaces $S$ cusps and the components of $M-U S$ pieces of $M$. The decomposition is proper if the set of cusps is nonempty. We shall also say that a manifold virtually has some property if it has a finite covering space with that property.

THEOREM 1. If a closed 4-manifold $\boldsymbol{M}$ admits a geometric decomposition then either

(i) $M$ is geometric; or

(ii) $M$ has a 2-fold cover which is an $S^{2} \times H^{2}$-manifold; or

(iii) the components of $M-\cup S$ have geometry $H^{4}, H^{3} \times E^{1}, H^{2} \times E^{2}$ or $\tilde{S L} \times E^{1}$; or

(iv) the components of $M-\cup S$ have geometry $H^{2}(C)$ or $F^{4}$; or

(v) the components of $M-\cup S$ all have geometry $H^{2} \times H^{2}$.

In cases (iii), (iv) or (v) $\chi(M) \geq 0$ and in cases (iii) or (iv) $M$ is aspherical.

Proof. The proof consists in considering the possible ends (cusps) of complete geometric 4-manifolds of finite volume. The hypersurfaces bounding a component of $M$ - US correspond to the ends of its interior. If the geometry is of solvable or compact type then there are no ends, since every lattice is then cocompact [Rg]. Thus we may concentrate on the eight geometries $S^{2} \times H^{2}, H^{2} \times E^{2}, H^{2} \times H^{2}, \tilde{S L} \times E^{1}$, $H^{3} \times E^{1}, H^{4}, H^{2}(C)$ and $F^{4}$. The ends of a geometry of constant negative curvature $H^{n}$ are flat [Eb80]; since any lattice in a Lie group must meet the radical in a lattice it follows easily that the ends are also flat in the mixed euclidean cases $H^{3} \times E^{1}, H^{2} \times E^{2}$ and $\tilde{S L} \times E^{1}$. Similarly, the ends of $S^{2} \times H^{2}$-manifolds are $S^{2} \times E^{1}$-manifolds. Since the elements of $\operatorname{PSL}(2, C)$ corresponding to the cusps of finite area hyperbolic surfaces are parabolic, the ends of $F^{4}$-manifolds are $N i l^{3}$-manifolds. The ends of $H^{2}(C)$ manifolds are also $\mathrm{Nil}^{3}$-manifolds [Ep87], while the ends of $\mathrm{H}^{2} \times \mathrm{H}^{2}$-manifolds are $\mathrm{Sol}^{3}$ manifolds, in the irreducible cases [Sh63], and graph manifolds whose fundamental groups contain nonabelian free subgroups otherwise. Clearly if two pieces are contiguous their common cusps must be homeomorphic. In all cases except when a piece is finitely covered by a product of two punctured hyperbolic surfaces the inclusion of a cusp into the closure of a piece induces a monomorphism on fundamental group.

If $M$ is a closed 4-manifold with a geometric decomposition of type (ii) the inclusions of the cusps into the closures of the pieces induce isomorphisms on $\pi_{2}$, and a Mayer-Vietoris argument in the universal covering space $\tilde{M}$ shows that $\tilde{M}$ is homotopy equivalent to $S^{2}$. Let $\kappa$ be the subgroup of $\pi_{1}(M)$ which acts trivially on $\pi_{2}(M) \cong Z$. Then $\left[\pi_{1}(M): \kappa\right] \leq 2$, and the cusps of the corresponding covering space $M_{\kappa}$ are copies of $S^{2} \times S^{1}$. Since the bundle projection to $S^{1}$ is unique up to isotopy and change of orientation of $S^{1}$ it follows that $M_{\kappa}$ admits a natural codimension-2 foliation with leaves 
$S^{2}$. Since the leaves $S^{2}$ are compact and 1-connected the projection to the leaf space is a submersion and $M_{\kappa}$ is the total space of an $S^{2}$-bundle over a hyperbolic surface. Hence $M_{\kappa}$ is an $S^{2} \times H^{2}$-manifold [CH96].

If at least one piece has an aspherical geometry other than $H^{2} \times H^{2}$ then all do and $M$ is aspherical. Since all the pieces of type $H^{4}, H^{2}(C)$ or $H^{2} \times H^{2}$ have strictly positive Euler characteristic while those of type $H^{3} \times E^{1}, H^{2} \times E^{2}, \tilde{S L} \times E^{1}$ or $F^{4}$ have Euler characteristic 0 we must have $\chi(M) \geq 0$ in cases (iii), (iv) or (v). //

In particular, if an aspherical closed 4-manifold has a nontrivial geometric decomposition with no pieces of type $H^{2} \times H^{2}$ then its fundamental group contains nilpotent subgroups of Hirsch length 3, corresponding to the cusps. (The Hirsch length of a solvable group is the sum of the ranks of the abelian subquotients in a composition series for the group).

If a closed 4-manifold admits a geometric decomposition, is there an essentially unique minimal decomposition? Since hyperbolic surfaces are connected sums of tori, and a punctured torus also admits a complete hyperbolic geometry of finite area, we cannot expect that there is an unique decomposition, even in dimension 2 . Any $P D_{n^{-}}$ group satisfying $\max -c$ (the maximal condition on centralizers) has an essentially unique minimal finite splitting along virtually poly- $Z$ subgroups of Hirsch length $n-1$, by Theorem A2 of [Kr90]. Do all fundamental groups of manifolds with geometric decompositions have max-c? Scott has announced an analogue of the 3-dimensional Characteristic Variety Theorem of Jaco, Shalen and Johanssen for higher-dimensional manifolds with metrics of non-positive sectional curvature [Sc92]. Do all closed 4manifolds of types (iii-v) admit such metrics?

Each of the four $S^{2} \times E^{1}$-manifolds inherits a natural codimension-1 foliation with compact leaves. It can be shown that these foliations are unique up to isotopy, and therefore every closed 4-manifold with a geometric decomposition of type (ii) admits a codimension-2 foliation with leaves $S^{2}$ or $R P^{2}$. If all the leaves are $R P^{2}$ such a manifold is the total space of an $R P^{2}$-bundle over a hyperbolic surface, and hence is geometric $[\mathbf{C H}]$. Otherwise it is the total space of an orbifold bundle over a hyperbolic 2-orbifold, with general fibre $S^{2}$. Are these also geometric?

Closed $H^{4}$ - or $H^{2}(C)$-manifolds admit no proper geometric decompositions, since their fundamental groups have no noncyclic abelian subgroups [Pr43]. A similar argument shows that closed $H^{3} \times E^{1}$-manifolds admit no proper decompositions, since they are finitely covered by cartesian products of $H^{3}$-manifolds with $S^{1}$. Thus closed 4manifolds with a proper geometric decomposition involving pieces of types other than $S^{2} \times H^{2}, H^{2} \times H^{2}, H^{2} \times E^{2}$ or $\tilde{S L} \times E^{1}$ are never geometric.

Many $S^{2} \times H^{2}, H^{2} \times H^{2}$ and $H^{2} \times E^{2}$-manifolds do admit proper geometric decompositions. (What is the case for $\tilde{S L} \times E^{1}$-manifolds?) On the other hand, a manifold with a geometric decomposition into pieces of type $H^{2} \times E^{2}$ need not be geometric. For instance, let $G=\langle u, v, x, y \mid[u, v]=[x, y]\rangle$ be the fundamental group of $T \sharp T$, the closed orientable surface of genus 2 , and let $\theta: G \rightarrow S L(2, Z)$ be the epi- 
morphism determined by $\theta(u)=\left(\begin{array}{cc}0 & -1 \\ 1 & 0\end{array}\right), \theta(x)=\left(\begin{array}{cc}0 & 1 \\ -1 & 1\end{array}\right)$ and $\theta(v)=\theta(y)=I$. Then the semidirect product $\pi=Z^{2} \times{ }_{\theta} G$ is the fundamental group of a torus bundle over $T \sharp T$ which has a geometric decomposition into two pieces of type $H^{2} \times E^{2}$, but is not geometric, since $\pi$ does not have a subgroup of finite index with centre isomorphic to $Z^{2}$.

Similarly, a manifold with a geometric decomposition into pieces of type $\mathrm{H}^{2} \times \mathrm{H}^{2}$ need be neither geometric nor aspherical. For instance, let $M$ be the double of $T_{o} \times T_{o}$, where $T_{o}=T-\operatorname{int} D^{2}$ is the once-punctured torus. Since $T_{o}$ admits a complete hyperbolic geometry of finite area $M$ admits a geometric decomposition into two pieces of type $\mathrm{H}^{2} \times \mathrm{H}^{2}$. However the homomorphism of fundamental groups induced by the inclusion of the cusp into $T_{o} \times T_{o}$ has nontrivial kernel, and $M$ is not aspherical.

It is easily seen that each $S^{2} \times E^{1}$-manifold may be realized as the end of a complete $S^{2} \times H^{2}$-manifold with finite volume and a single end. (However, if the manifold is orientable the ends must be orientable, and if it is complex analytic then they must be $\left.S^{2} \times S^{1}\right)$. Every flat 3-manifold is a cusp of some complete $H^{4}$-manifold with finite volume [Ni95]. However the fundamental group of a cusp of an $\tilde{S L} \times E^{1}$-manifold must have a chain of abelian normal subgroups $Z<Z^{2}<Z^{3}$, and so the only orientable flat 3-manifolds that can arise in this way are the 3-torus, the "half-turn" manifold and the Hantzsche-Wendt manifold (with fundamental groups $Z^{3}, Z^{2} \times_{-I} Z$ and $\left\langle x, y \mid x y^{2} x^{-1}=y^{-2}, y x^{2} y^{-1}=x^{-2}\right\rangle$, respectively). The ends of complete, complex analytic $\mathrm{H}^{2} \times \mathrm{H}^{2}$-manifolds with finite volume are orientable $\mathrm{Sol}^{3}$-manifolds which are mapping tori, and all such may be realized in this way [Sh63].

\section{§2. Seifert fibrations.}

A closed 4-manifold is Seifert fibred if it is the total space of an orbifold bundle with base a 2-orbifold and general fibre a torus or Klein bottle. (In [Zi85], [Ue90,91] it is required that the general fibre be a torus. This is always so if the manifold is orientable). The fundamental group $\pi$ of such a 4-manifold has a rank two free abelian normal subgroup $A$ such that $\pi / A$ is virtually a surface group. Every Seifert fibred 4manifold is finitely covered by the total space of a torus bundle over a closed surface. (This is clear if the base orbifold is good, and follows from Theorem A of [Ue91] in the remaining cases). The results of this section imply that (with two exceptions which are flat 4-manifolds) orientable quotients of torus bundles over aspherical surfaces by finite groups are at least homotopy equivalent to Seifert fibred manifolds.

THEOREM 2. Let $M$ be a closed 4-manifold with fundamental group $\pi$. Then $M$ is simple homotopy equivalent to the total space of a torus or Klein bottle bundle over an aspherical closed surface if and only if $\pi$ has a normal subgroup $A \cong Z^{2}$ or $Z \tilde{\times} Z$ such that $\pi / A$ is torsion free and has infinite abelianization and $\chi(M)=0$.

Proof. The conditions are clearly necessary. Suppose that they hold. Then $M$ is aspherical, so $\pi$ is a $P D_{4}$-group. It follows from the LHS spectral sequence that $H^{2}(\pi / A ; R[\pi / A]) \cong H^{4}(\pi ; R[\pi]) \cong R$ for $R=Z$ or a field. Since $\pi / A$ is torsion free and 
has infinite abelianization it has cohomological dimension $\leq 3$, by Corollary 2.3 of [Fa74], and so is a $P D_{2}$-group [Fa75]. Thus $M$ is homotopy equivalent to the total space of a $K(A, 1)$-bundle over $K(\pi / A, 1)$. The homotopy equivalence is simple by Lemma V.2 of $[\mathbf{H}]$. //

Every Seifert fibred 4-manifold with base an euclidean orbifold has Euler characteristic 0 and fundamental group solvable of Hirsch length 4, and so is homeomorphic to an infrasolvmanifold, by Theorem VI.2 of $[\mathbf{H}]$. It is easily seen that no group of type $\mathrm{Sol}_{0}^{4}, \mathrm{Sol}_{1}^{4}$ or $\mathrm{Sol}_{m, n}^{4}$ (with $m \neq n$ ) has a rank two free abelian normal subgroup. Hence the manifold must have one of the geometries $E^{4}, N i l \times E^{1}, S o l \times E^{1}$ or $N i l^{4}$. Conversely, excepting only three flat 4-manifolds, such manifolds are Seifert fibred. The fundamental group of a closed $\mathrm{Nil}^{3} \times \mathrm{E}^{1}$ - or $\mathrm{Nil}^{4}$-manifold has a rank two free abelian normal subgroup, by Theorem VI.3 of $[\mathbf{H}]$. If $\pi$ is the fundamental group of a $\mathrm{Sol}^{3} \times E^{1}$-manifold then the commutator subgroup of the intersection of all index 4 subgroups is such a subgroup. (In the $\mathrm{Nil}^{4}$ and $\mathrm{Sol}^{3} \times E^{1}$ cases there is an unique maximal such subgroup). Case-by-case inspection of the 74 flat 4-manifold groups shows that all but three have such subgroups [Hi95]. (Ue actually shows that in the orientable cases such Seifert fibred 4-manifolds are diffeomorphic to such infrasolvmanifolds, and the Seifert fibration is unique up to diffeomorphism, which may be assumed fibre-preserving in the $\mathrm{Nil}^{4}$ and $\mathrm{Sol}^{3} \times E^{1}$ cases. There is a minor oversight in [Ue90]; in fact there are two orientable flat four-manifolds which are mapping tori of homeomorphisms of the Hantszche-Wendt flat 3-manifold and which are not Seifert fibred.

Orientable Seifert fibred 4-manifolds with base a spherical or bad orbifold admit one of the geometries $S^{2} \times E^{2}$ or $S^{3} \times E^{1}$, and conversely every such geometric 4-manifold is Seifert fibred over such a base, although in general it may admit many distinct Seifert fibrations [Ue91]. (See also [Oh90] for some of the nonorientable cases). The homotopy type of a $S^{2} \times E^{2}$-manifold is determined up to finite ambiguity by the fundamental group (which must be virtually $Z^{2}$ ), Euler characteristic (which must be 0 ) and Stiefel-Whitney classes. There are just nine possible fundamental groups. Six of these have infinite abelianization, and the above invariants determine the homotopy type in these cases. (See Chapter VII of $[\mathbf{H}]$ ). The homotopy type of a $S^{3} \times E^{1}$-manifold is determined by the fundamental group (which must be virtually $Z$ ), Euler characteristic (which must be 0 ), orientation character $w_{1}$ and first $k$-invariant in $H^{4}\left(\pi ; \pi_{3}\right)$. (See Chapter VIII of $[\mathbf{H}])$.

If $\pi<O(4) \times E(1)$ is the fundamental group of an $S^{3} \times E^{1}$-manifold then $F=$ $\pi \cap S O(4) \times\{1\}$ is the maximal finite normal subgroup of $\pi$ and acts freely on $S^{3}$, and $\pi / F \cong Z$ or $D=(Z / 2 Z) *(Z / 2 Z)$. In the first case $\pi$ is a semidirect product $F \times_{\theta} Z$, where $\theta$ is an automorphism of $F$ induced by an isometry of $S^{3} / F$ and in the second case $\pi \cong G *_{F} H$, where the projections of $G$ and $H$ to $O(4)$ are injective, with images acting freely on $S^{3}$, and $[G: F]=[H: F]=2$. In particular, the double covers of the spherical space forms $G \backslash S^{3}$ and $H \backslash S^{3}$ corresponding to the subgroup $F$ must be 
homeomorphic. Conversely, if $G$ and $H$ act freely and linearly on $S^{3}$ and have subgroups of index 2 isomorphic to $F$ and if either $G$ or $H$ is cyclic or $F$ is not cyclic then $G *_{F} H$ is the fundamental group of an $S^{3} \times E^{1}$-manifold.

Example. The groups $G=Q(40)$ and $H=Q(8) \times Z / 5 Z$ correspond to spherical space forms doubly covered by the lens spaces $L(20,1)$ and $L(20,9)$, respectively, which are homotopy equivalent but not homeomorphic. Thus $G *_{F} H$ is realized by the union of two twisted I-bundles via a homotopy equivalence, which is a finite (but possibly nonsimple?) $P D_{4}$-complex with $\chi=0$. If $\pi$ is realized by a finite simple $P D_{4}$-complex with $\chi=0$ must it be geometric? The groups $G=Q(24)$ and $H=Q(8) \times Z / 3 Z$ correspond to spherical space forms doubly covered by $L(12,1)$ and $L(12,5)$, respectively, which are not even homotopy equivalent.

A manifold with geometry $H^{2} \times E^{2}$ or $\tilde{S L} \times E^{1}$ is Seifert fibred with base a hyperbolic orbifold. However not all such Seifert fibred 4-manifolds are geometric. An orientable Seifert fibred 4-manifold over an orientable hyperbolic base is geometric if and only if it is an elliptic surface; the relevant geometries are then $H^{2} \times E^{2}$ and $\tilde{S L} \times E^{1}[$ Ue90,91]

If $\pi$ is a group $\pi^{\prime}, \zeta \pi$ and $\sqrt{\pi}$ are its commutator subgroup, centre and HirschPlotkin radical, respectively. If $G \leq \pi$ is a subgroup then $N_{\pi}(G)$ and $C_{\pi}(G)$ are the normalizer and centralizer of $G$ in $\pi$, respectively.

THEOREM 3. Let $M$ be a closed orientable 4-manifold with fundamental group $\pi$. Then $M$ is homotopy equivalent to a manifold which is Seifert fibred over a hyperbolic orbifold if and only if $\sqrt{\pi} \cong Z^{2}, G=\pi / \sqrt{\pi}$ has a subgroup of finite index which is torsion free and has infinite abelianization and $\chi(M)=0$.

Proof. The conditions are clearly necessary. Suppose that they hold. Then $M$ is aspherical, so $\pi$ is a $P D_{4}$-group. It follows as in Theorem 2 that $H^{2}(G ; Z[G]) \cong Z$ and hence that $G$ is virtually a $P D_{2}$-group. If $F$ is a finite normal subgroup of $G$ then the preimage of $F$ in $\pi$ is torsion free and virtually abelian. Since $M$ is orientable this preimage must be abelian and so $G$ has no nontrivial finite normal subgroup. Moreover $G$ is not virtually abelian, for otherwise $\pi$ would be virtually solvable and hence $h(\sqrt{\pi}) \geq 3$. Therefore $G$ acts effectively and properly discontinuously on $H^{2}$, by the solution to the Nielsen realization problem for surfaces [Ke83]. Since $\pi$ is torsion free it is the fundamental group of a closed aspherical 4-manifold $M(\pi)$ which is Seifert fibred over $G \backslash H^{2}$ [CR72]. //

In particular, if $\sqrt{\pi}$ is central $\left(\zeta \pi \cong Z^{2}\right)$ then the corresponding Seifert fibered manifold $M(\pi)$ admits an effective torus action with finite isotropy subgroups. The LHS spectral sequence for homology gives an exact sequence $H_{2}(\pi / \zeta \pi ; Q) \rightarrow$ $H_{1}(\zeta \pi ; Q) \rightarrow H_{1}(\pi ; Q)$. Since $H_{2}(\pi / \zeta \pi ; Q) \cong Q$ or 0 it follows that $\zeta \pi / \zeta \pi \cap \pi^{\prime}$ has rank at least 1 . Hence $M(\pi)$ fibres over $S^{1}$ with monodromy of finite order. In these cases we have $W h(\pi)=0$ [NS85]. Is $M s$-cobordant to $M(\pi)$ ? 
COROLlARY. Let $M$ be a closed orientable 4-manifold with fundamental group $\pi$. Then $M$ is homotopy equivalent to a $\tilde{S L} \times E^{1}$-manifold or an $H^{2} \times E^{2}$-manifold if and only if $\sqrt{\pi} \cong Z^{2}, \pi / \sqrt{\pi}$ has a subgroup of finite index which is torsion free and has infinite abelianization, $\left[\pi: C_{\pi}(\sqrt{\pi})\right]<\infty$ and $\chi(M)=0$.

Proof. The necessity of the conditions is proven in Theorem VI.13 of $[\mathbf{H}]$. Suppose that they hold. Then $M$ is homotopy equivalent to a manifold $M(\pi)$ which is Seifert fibred over a hyperbolic base orbifold, and the image of $\pi$ in $\operatorname{Aut}(\sqrt{\pi}) \cong G L(2, Z)$ is a finite group. The result follows as in Theorem $B$ of $\$ 5$ of [Ue91]. //

The $H^{2} \times E^{2}$ case may be distinguished as follows.

THEOREM 4. Let $M$ be a closed orientable 4-manifold with fundamental group $\pi$. Then $M$ is homotopy equivalent to a closed $H^{2} \times E^{2}$-manifold if and only if $\pi$ has a finitely generated subgroup $\rho$ such that $\rho / \rho^{\prime}$ is infinite, $\sqrt{\rho}=1,\left[\pi: N_{\pi}(\rho)\right]<\infty$, $\zeta C_{\pi}(\rho) \cong Z^{2}$ and $\chi(M)=0$.

Proof. The necessity of the conditions follows from Theorem VI.13 of $[\mathbf{H}]$. Suppose that they hold. Then $M$ is aspherical and so $\pi$ is a $P D_{4}$-group. Let $C=C_{\pi}(\rho)$. Then $C$ is also normal in $v=N_{\pi}(\rho)$, and $C \cap \rho=1$, since $\sqrt{\rho}=1$. Hence $\rho \times C \cong \rho . C \leq \pi$. Now $\rho$ is nontrivial. If $\rho$ were free then an argument using the LHS spectral sequence for $H^{*}(\pi ; Q[\pi])$ would imply that $\rho$ has two ends, and hence that $\sqrt{\rho}=\rho \cong Z$. Hence $c . d . \rho \geq 2$. Since moreover $Z^{2} \leq C$ we must have c.d. $\rho=$ c.d.C $=$ 2 and $[\pi: \rho . C]<\infty$. Hence $\rho$ is a $P D_{2}$-group of hyperbolic type.

Since $\zeta v \cong Z^{2}$ it follows easily that $\sqrt{\pi} \cong Z^{2}$ and that $\left[\pi: C_{\pi}(\sqrt{\pi})\right]<\infty$. The image of $\rho$ in $\pi / \sqrt{\pi}$ is torsion free and of finite index. Hence the conditions of the above Corollary hold. Since $\pi$ is virtually a product it must be of type $H^{2} \times E^{2}$. //

Is it possible to give a more self-contained argument for this case? It is not hard to see that $\pi / \sqrt{\pi}$ acts isometrically and properly discontinuously on $H^{2}$. However it is more difficult to construct a suitable homomorphism from $\pi$ to $\operatorname{Isom}\left(E^{2}\right)$.

The closed 4-manifold obtained by surgery on a twist spun torus knot in general admits the geometry $\tilde{S L} \times E^{1}$. (See Chapter $\mathrm{X}$ of $[\mathbf{H}]$ ). Every orientable $H^{2} \times E^{2}$ manifold is double covered by a Kähler surface [W186]. Since the unique double cover of a 2-knot manifold $M(K)$ has first Betti number 1, no such manifold can be an $H^{2} \times E^{2}$-manifold. Can such a knot manifold be Seifert fibred but not geometric?

EXAMPLES. If $M$ is a compact manifold with boundary whose interior is an $F^{4}$ manifold of finite volume then $\pi_{1}(M)$ is a semidirect product $Z^{2} \times_{\theta} F$ where $\theta: F \rightarrow$ $G L(2, Z)$ is a monomorphism with image of finite index. The double $D M=M \cup_{\partial} M$ is Seifert fibred over a hyperbolic base but is not geometric, since $\sqrt{\pi} \cong Z^{2}$ but $\left[\pi: C_{\pi}(\sqrt{\pi})\right]$ is infinite. The orientable surface of genus 2 can be represented as a double in two distinct ways; we shall give corresponding examples of nongeometric torus bundles which admit geometric decompositions of type $F^{4}$. (Note that $F^{4}$-manifolds are Seifert fibred with base a punctured hyperbolic orbifold). 
1. Let $F(2)$ be the free group of rank two and let $\gamma: F(2) \rightarrow S L(2, Z)$ have image the commutator subgroup $S L(2, Z)^{\prime}$, which is freely generated by $A=\left(\begin{array}{cc}0 & -1 \\ 1 & 3\end{array}\right)$ and $B=\left(\begin{array}{ll}1 & 1 \\ 1 & 2\end{array}\right)$. The natural surjection from $S L(2, Z)$ to $\operatorname{PSL}(2, Z)$ induces an isomorphism of commutator subgroups, and the parabolic subgroup $\operatorname{PSL}(2, Z)^{\prime} \cap \operatorname{Stab}(0)$ is generated by the image of $A B^{-1} A^{-1} B=\left(\begin{array}{cc}-1 & 0 \\ -6 & -1\end{array}\right)$. Hence $\left[\operatorname{Stab}(0): P S L(2, Z)^{\prime} \cap \operatorname{Stab}(0)\right]=6=$ $\left[P S L(2, Z): P S L(2, Z)^{\prime}\right]$, and so $P S L(2, Z)^{\prime}$ has a single cusp at 0 . The quotient space $\operatorname{PSL}(2, Z)^{\prime} \backslash H^{2}$ is the once-punctured torus. Let $N \subset P S L(2, Z)^{\prime} \backslash H^{2}$ be the complement of an open horocyclic neighbourhood of the cusp. The double $D N$ is the closed orientable surface of genus 2. The semidirect product $\Gamma=Z^{2} \times{ }_{\gamma} F(2)$ is a lattice in Isom $\left(F^{4}\right)$, and the double of the bounded manifold with interior $\Gamma \backslash F^{4}$ is a torus bundle over $D N$.

2. Let $\delta: F(2) \rightarrow S L(2, Z)$ have image the subgroup which is freely generated by $U=\left(\begin{array}{ll}1 & 0 \\ 2 & 1\end{array}\right)$ and $V=\left(\begin{array}{ll}1 & 2 \\ 0 & 1\end{array}\right)$. Let $\bar{\delta}: F(2) \rightarrow P S L(2, Z)$ be the composed map. Then $\bar{\delta}$ is injective and $[P S L(2, Z): \bar{\delta}(F(2))]=6$. (Note that $\delta(F(2))$ and $-I$ together generate the level 2 congruence subgroup). Moreover $[\operatorname{Stab}(0): \bar{\delta}(F(2)) \cap \operatorname{Stab}(0)]=2$. Hence $\bar{\delta}(F(2))$ has three cusps, at $0, \infty$ and 1 , and $\bar{\delta}(F(2)) \backslash H^{2}$ is the thrice-punctured sphere. The corresponding parabolic subgroups are generated by $U, V$ and $V U^{-1}$, respectively. Doubling the complement $N$ of disjoint horocyclic neighbourhoods of the cusps in $\bar{\delta}(F(2)) \backslash H^{2}$ again gives a closed orientable surface of genus 2. (The presentation for $\pi_{1}(D N)$ derived from this construction is $\left\langle U, V, U_{1}, V_{1}, s, t\right| s^{-1} U s=$ $\left.U_{1}, \quad t^{-1} V t=V_{1}, V U^{-1}=V_{1} U_{1}^{-1}\right\rangle$, which is equivalent to the usual presentation $\left.\left\langle U, V, s, t \mid s^{-1} V^{-1} s V=t^{-1} U^{-1} t U\right\rangle\right)$. The semidirect product $\Delta=Z^{2} \times_{\delta} F(2)$ is a lattice in $\operatorname{Isom}\left(F^{4}\right)$, and the double of the bounded manifold with interior $\Delta \backslash F^{4}$ is again a torus bundle over $D N$.

3. If $G$ is an orientable $P D_{2}$-group which is not virtually $Z^{2}$ and $\lambda: G \rightarrow S L(2, Z)$ is a homomorphism whose image is infinite cyclic then $\pi=Z^{2} \times_{\lambda} G$ is the fundamental group of a closed orientable 4-manifold which is Seifert fibred over an orientable hyperbolic surface but which has no geometric decomposition at all. (The only possible geometries are $F^{4}, H^{2} \times E^{2}$ and $\tilde{S L} \times E^{1}$. We may exclude pieces of type $F^{4}$ as $\operatorname{Im}(\lambda)$ has infinite index in $S L(2, Z)$, and we may exclude pieces of type $H^{2} \times E^{2}$ or $\tilde{S L} \times E^{1}$ as $\operatorname{Im}(\lambda) \cong Z$ is not generated by finite subgroups).

\section{§3. Virtual products.}

In this section we give criteria for a closed 4-manifold to be (homotopy equivalent to one which is) virtually a cartesian product of closed hyperbolic surfaces. Such products are clearly geometric.

Let $P=P S L(2, R)$ be the group of orientation preserving isometries of $H^{2}$. Then Isom $\left(H^{2} \times H^{2}\right)$ contains $P \times P$ as a normal subgroup of index 8 .

THEOREM 5. Let $M$ be a closed 4-manifold with fundamental group $\pi$. Then the following are equivalent: 
(i) $M$ is virtually homotopy equivalent to a cartesian product of two hyperbolic surfaces;

(ii) $\pi_{2}(M)=0, \chi(M) \neq 0$ and $\pi=\pi_{1}(M)$ has a subnormal subgroup $G$ which is $F P_{2}$, has one end and such that $C_{\pi}(G)$ is not a free group;

(iii) $\pi_{2}(M)=0, \chi(M) \neq 0$ and $\pi$ has a subgroup $G$ which is a $P D_{2}$-group such that $\left[\pi: N_{\pi}(G)\right] \leq 2$ and $C_{\pi}(G)$ is not a free group;

(iv) $M$ is homotopy equivalent to an $\mathrm{H}^{2} \times \mathrm{H}^{2}$-manifold with a 2-fold covering space which is the total space of an orbifold bundle over a hyperbolic base 2-orbifold and with general fibre a hyperbolic surface;

(v) $\pi$ has a subgroup $\rho$ of finite index which is isomorphic to a product of two $P D_{2-}$ groups and $\chi(M)[\pi: \rho]=\chi(\rho) \neq 0$

(vi) $\pi$ is virtually a $P D_{4}$-group, $\sqrt{\pi}=1$ and $\pi$ has a torsion free normal subgroup of finite index which is isomorphic to a nontrivial product $\sigma \times \tau$ where $\chi(M)[\pi: \sigma \times \tau]=$ $\left(2-\beta_{1}(\sigma)\right)\left(2-\beta_{1}(\tau)\right)$.

Proof. If (i) holds then $M$ is aspherical so $\pi_{2}(M)=0$, and $\pi$ has a subgroup of finite index which is a direct product $\alpha . \beta \cong \alpha \times \beta$, where $\alpha$ and $\beta$ are $P D_{2}$-groups and $\alpha \cap \beta=\zeta \alpha=1$. Since $\chi(M)=\chi(\pi)$ divides $\chi(\alpha) \chi(\beta)$ it is nonzero. Let $N$ be the intersection of the conjugates of $\alpha . \beta$ in $\pi$. Then $G=N \cap \alpha$ is subnormal in $\pi$ and $[\alpha: G] \leq[\pi: N]<\infty$. Hence $G$ is the fundamental group of a hyperbolic surface and so is $F P_{2}$ and one-ended. Moreover $\beta \leq C_{\pi}(G)$ and so c.d. $C_{\pi}(G) \geq 2$. Thus (i) implies (ii).

Suppose now that (ii) holds. Then $\pi$ has one end, by an iterated LHS spectral sequence argument, since $G$ does. Hence $M$ is aspherical and $\pi$ is a $P D_{4}$-group, since $\pi_{2}(M)=0$. Since $\chi(M) \neq 0$ we must have $\sqrt{\pi}=1$, by the corollary to Theorem II. 6 of $[\mathbf{H}]$. In particular, every subnormal subgroup of $\pi$ has trivial centre. Therefore $G \cap C_{\pi}(G)=\zeta G=1$ and so $G \times C_{\pi}(G) \cong \rho=G . C_{\pi}(G) \leq \pi$. Hence c.d. $C_{\pi}(G) \leq 2$. Since $C_{\pi}(G)$ is not free c.d. $G \times C_{\pi}(G)=4$ and so $\rho$ has finite index in $\pi$. (In particular, $\left[C_{\pi}\left(C_{\pi}(G)\right): G\right]$ is finite). Hence $\rho$ is a $P D_{4}$-group and $G$ and $C_{\pi}(G)$ are $P D_{2}$-groups. Let $\sigma$ be the intersection of the conjugates of $\rho$. Then $\sigma$ is normal in $\pi$ and $[\pi: \sigma]<\infty$. Hence $K=G \cap \sigma$ and $L=C_{\pi}(G) \cap \sigma$ are also $P D_{2}$-groups. They are normal in $\sigma$, and the quotients $\sigma / K$ and $\sigma / L$ are virtually $P D_{2}$-groups. Let $\hat{K}$ and $\hat{L}$ be the preimages in $\sigma$ of the maximal finite normal subgroups of $\sigma / K$ and $\sigma / L$, respectively. Then $\hat{K}$ and $\hat{L}$ are also $P D_{2}$-groups with trivial centre, and $\hat{K} \cap \hat{L}=1$. Hence $\hat{K} \cdot \hat{L} \cong \hat{K} \times \hat{L}$.

Suppose that $N$ is another normal subgroup of $\sigma$ which is a $P D_{2}$-subgroup and such that $\sigma / N$ is virtually a $P D_{2}$-group and has no nontrivial finite normal subgroup. Let $H=N \cap(\hat{K} . \hat{L})$. Since $H$ is normal in $\sigma$ it contains the subgroups $[H, \hat{K}]$ and $[H, \hat{L}]$ and hence their product $[H, \hat{K}] .[H, \hat{L}]$. Since $H$ is a $P D_{2}$-group with trivial centre one of these factors must be trivial. If $[H, \hat{K}]=1$ then $H \leq \hat{L}$ and so $N$ is commensurate with $\hat{L}$; since neither $\sigma / \hat{L}$ nor $\sigma / N$ have nontrivial finite normal subgroups it follows that $N=\hat{L}$. (This argument to show that $N=\hat{K}$ or $\hat{L}$ is based on the proof of 
Theorem B of [Jo94]). Since conjugation by an element of $\pi$ carries $\hat{K}$ onto another such subgroup it follows that $\left[\pi: N_{\pi}(\hat{K})\right] \leq 2$. (Similarly, $\hat{K} . \hat{L}$ is normal in $\pi$ ). Thus (ii) implies (iii). (Note that (iii) clearly implies (iii)).

Suppose now that (iii) holds, and let $v=N_{\pi}(G)$. We may assume that $v / G$ has no nontrivial finite normal subgroup. If $G$ is normal in $\pi$ then so is $C_{\pi}(G)$, and the action of $\pi / C_{\pi}(G)$ by conjugation on $G$ has finite image in $\operatorname{Out}(G)$. Therefore $\pi / C_{\pi}(G)$ embeds as a discrete cocompact subgroup of $\operatorname{Isom}\left(H^{2}\right)$, by the Nielsen conjecture [Ke83]. Since $\left[C_{\pi}\left(C_{\pi}(G)\right): G\right]$ is finite it follows that $C_{\pi}\left(C_{\pi}(G)\right)=G$, and so $\pi / G=$ $\pi / C_{\pi}\left(C_{\pi}(G)\right)$ also embeds as a discrete cocompact subgroup of $\operatorname{Isom}\left(H^{2}\right)$. Together these homomorphisms give a homomorphism from $\pi$ to a discrete cocompact subgroup of $\operatorname{Isom}\left(H^{2} \times H^{2}\right)$.

If $[\pi: v]=2$ let $t$ be an element of $\pi-v$, and let $j: v / G \rightarrow \operatorname{Isom}\left(H^{2}\right)$ be an embedding onto a discrete cocompact subgroup $S$. Then $t G t^{-1}=C_{\pi}(G)$ and $t$ induces an isomorphism $f: v / G \rightarrow v / C_{\pi}(G)$. Together $j$ and $j \circ f^{-1}$ determine an embedding $J: v \rightarrow \operatorname{Isom}\left(H^{2} \times H^{2}\right)$ onto a discrete cocompact subgroup of finite index in $S \times S$. Now $t^{2} \in v$ and $J\left(t^{2}\right)=(s, s)$, where $s=j\left(t^{2} G\right)$. We may extend $J$ to an embedding of $\pi$ in $\operatorname{Isom}\left(H^{2} \times H^{2}\right)$ by defining $J(t)$ to be the isometry sending $(x, y)$ to $(y, s . x)$. Thus $\pi$ acts isometrically and properly discontinuously on $H^{2} \times H^{2}$. Since $\pi$ is torsion free the action is free, and so $M$ is homotopy equivalent to the quotient manifold $\pi \backslash\left(H^{2} \times H^{2}\right)$. Thus (iii) implies (iv).

An irreducible lattice in $P \times P$ cannot have any nontrivial normal subgroups of infinite index, by Theorem IX.6.14 of [M]. Hence an $H^{2} \times H^{2}$-manifold which is finitely covered by the total space of a surface bundle is virtually a cartesian product and so (iv) implies (i).

It is clear that (i) implies (v), and (v) implies (vi). (Note that we may assume there is a normal subgroup of this form, by the argument above). Suppose that (vi) holds. On replacing $\pi$ by a subgroup of finite index, if necessary, we may assume that $\pi \cong \sigma \times \tau$. Since $\sqrt{\pi}=1$ neither factor can be infinite cyclic, and so $\sigma$ and $\tau$ are each $P D_{2}$-groups. Since $\sqrt{\sigma}=\sqrt{\tau}=1$, these groups are fundamental groups of closed orientable $H^{2}$-manifolds, $F_{\sigma}$ and $F_{\tau}$, say. It now follows from Theorem II.5 of $[\mathbf{H}]$ that $M$ is homotopy equivalent to $F_{\sigma} \times F_{\tau}$, which is clearly an $H^{2} \times H^{2}$ manifold. //

The asphericity of $M$ could be ensured by assuming that $\pi$ be $P D_{4}$ and $\chi(M)=\chi(\pi)$, instead of assuming that $\pi_{2}(M)=0$.

In general, we cannot assume that $M$ is itself fibred over a 2-orbifold. Let $G$ be a $P D_{2}$-group with $\zeta G=1$ and let $x$ be a nontrivial element of $G$. A uniform free action of $G$ on $H^{2}$ determines a uniform free action of $\pi=\langle G \times G, t| t\left(g_{1}, g_{2}\right) t^{-1}=$ $\left.\left(x g_{2} x^{-1}, g_{1}\right) \forall\left(g_{1}, g_{2}\right) \in G \times G, t^{2}=(x, x)\right\rangle$ on $H^{2} \times H^{2}$, by $\left(g_{1}, g_{2}\right) \cdot\left(h_{1}, h_{2}\right)=\left(g_{1} \cdot h_{1}, g_{2} \cdot h_{2}\right)$ and $t .\left(h_{1}, h_{2}\right)=\left(x . h_{2}, h_{1}\right)$, for all $\left(g_{1}, g_{2}\right) \in G \times G$ and $\left(h_{1}, h_{2}\right) \in H^{2} \times H^{2}$. The group $\pi$ has no normal subgroup which is a $P D_{2}$-group. (Note also that if $G$ is orientable $\pi \backslash\left(H^{2} \times H^{2}\right)$ is a compact complex surface). 
COROLlARY. If $M$ is an aspherical closed 4-manifold which is virtually geometric then $M$ is homotopy equivalent to a geometric 4-manifold. If the geometry is $H^{2} \times E^{2}$ or $\tilde{S L} \times E^{1}$ we should assume also that $M$ is orientable.

Proof. The result is clear for infrasolvmanifolds, and follows from the characterizations in Theorems 3 and 4 above for closed orientable $H^{2} \times E^{2}$ - and $\tilde{S L} \times E^{1}$ manifolds. If $\pi$ is virtually the group of an $H^{3} \times E^{1}$-manifold then $\pi / \sqrt{\pi}$ is virtually the group of an $H^{3}$-manifold, and therefore acts isometrically on $H^{3}$ by Mostow rigidity. We may also find $\lambda: \pi \rightarrow Z$ or $D$ such that $\lambda(\sqrt{\pi})$ is nontrivial. Thus $\pi$ acts freely, cocompactly and isometrically on $H^{3} \times E^{1}$. Theorem 5 shows that the result is true if $M$ is finitely covered by the product of two closed hyperbolic surfaces. For the other closed $H^{2} \times H^{2}$-manifolds (with fundamental group an irreducible lattice) and for the geometries $H^{4}$ and $H^{2}(C)$ the result holds by Mostow rigidity. //

The corollary holds also for the geometries $S^{4}$ and $C P^{2}$, but is not yet clear for manifolds covered by $S^{2} \times S^{2}, S^{2} \times R^{2}$ or $S^{3} \times R$. In particular, there is a closed nonorientable 4-manifold which is doubly covered by $S^{2} \times S^{2}$ but is not geometric. (See Chapter IX of $[\mathbf{H}]$ ). However orientability is surely unnecessary for the geometries $H^{2} \times E^{2}$ and $\tilde{S L} \times E^{1}$.

For $H^{2} \times H^{2}$-manifolds we can give more precise criteria for virtual products.

THEOREM 6. Let $M$ be a closed $H^{2} \times H^{2}$-manifold with fundamental group $\pi$. Then the following are equivalent:

(i) $\pi$ has a subgroup of finite index which is a nontrivial direct product;

(ii) $Z^{2}<\pi$;

(iii) $\pi$ has a nontrivial element with nonabelian centralizer;

(iv) $\pi \cap(\{1\} \times P) \neq 1$;

(v) $\pi \cap(P \times\{1\}) \neq 1$;

(vi) $M$ is finitely covered by the cartesian product of two hyperbolic surfaces.

Proof. Since $\pi$ is torsion free each of the above conditions is invariant under passage to subgroups of finite index, and so we may assume without loss of generality that $\pi \leq P \times P$. Suppose that $\sigma$ is a subgroup of finite index in $\pi$ which is a nontrivial direct product. Since $\chi(\sigma) \neq 0$ neither factor can be infinite cyclic, and so the factors must be $P D_{2}$-groups. In particular, $Z^{2}<\sigma$ and the centralizer of any element of either direct factor is nonabelian. Thus (i) implies (ii) and (iii).

Suppose that $(a, b)$ and $\left(a^{\prime}, b^{\prime}\right)$ generate a subgroup of $\pi$ isomorphic to $Z^{2}$. Since centralizers of nontrivial elements of infinite order in $P$ are cyclic the subgroup of $P$ generated by $\left\{a, a^{\prime}\right\}$ is infinite cyclic or is finite. Hence we may assume without loss of generality that $a^{\prime}=1$, and so (ii) implies (iv). Similarly, (ii) implies (v).

Let $g=\left(g_{1}, g_{2}\right) \in P \times P$ be nontrivial. Since $C_{P \times P}(\langle g\rangle)=C_{P}\left(\left\langle g_{1}\right\rangle\right) \times C_{P}\left(\left\langle g_{2}\right\rangle\right)$ and centralizers of nontrivial elements of infinite order in $P$ are infinite cyclic it follows that if $C_{\pi}(\langle g\rangle)$ is nonabelian then either $g_{1}$ or $g_{2}$ has finite order. Thus (iii) implies (iv) or $(\mathbf{v})$. 
Let $K_{1}=\pi \cap(\{1\} \times P)$ and $K_{2}=\pi \cap(P \times\{1\})$. Then $K_{i}$ is normal in $\pi$, and there are exact sequences $1 \rightarrow K_{i} \rightarrow \pi \rightarrow L_{i} \rightarrow 1$, where $L_{i}=p r_{i}(\pi)$ is the image of $\pi$ under projection to the $i^{\text {th }}$ factor of $P \times P$, for $i=1$ and 2. Moreover $K_{i}$ is normalized by $L_{3-i}$, for $i=1$ and 2. Suppose that $K_{1} \neq 1$. Then $K_{1}$ is nonabelian, since it is normal in $\pi$ and $\chi(\pi) \neq 0$. If $L_{2}$ were not discrete then elements of $L_{2}$ sufficiently close to the identity would centralize $K_{1}$. As centralizers of nonidentity elements of $P$ are abelian, this would imply that $K_{1}$ is abelian. Hence $L_{2}$ is discrete. Now $L_{2} \backslash H^{2}$ is a quotient of $\pi \backslash H \times H$ and so is compact. Therefore $L_{2}$ is virtually a $P D_{2}$-group. Now c.d. $K_{2}+$ v.c.d. $L_{2} \geq$ c.d. $\pi=4$, so c.d. $K_{2} \geq 2$. In particular, $K_{2} \neq 1$ and so a similar argument now shows that c.d. $K_{1} \geq 2$. Hence c.d. $K_{1} \times K_{2} \geq 4$. Since $K_{1} \times K_{2} \cong$ $K_{1} \cdot K_{2} \leq \pi$ it follows that $\pi$ is virtually a product, and $M$ is finitely covered by $\left(K_{1} \backslash H^{2}\right) \times\left(K_{2} \backslash H^{2}\right)$. Thus (iv) and (v) are equivalent, and imply (vi).

Clearly (vi) implies (i). //

The idea used in showing that (iv) implies (v) and (vi) derives from one used in the proof of Theorem 6.3 of [W185].

\section{§4. Surface bundles and geometries.}

Let $p: E \rightarrow B$ be a bundle with base $B$ and fibre $F$ aspherical closed surfaces. Then $p$ is determined up to bundle isomorphism by the group $\pi=\pi_{1}(E)$. The cases with $\chi(F)=0$ are among the Seifert fibred 4-manifolds considered in $\S 2$, and so we shall assume that $F$ is hyperbolic, i.e. that $\chi(F)<0$. Then $\zeta \pi_{1}(F)=1$ and so $\pi$ is determined up to isomorphism by the characteristic homomorphism $\theta: \pi_{1}(B) \rightarrow$ $\operatorname{Out}\left(\pi_{1}(F)\right)$. (See Theorem IV.1 of $[\mathbf{H}]$ ). In this section we consider when an aspherical 4-manifold which is finitely covered by such a bundle space is geometric or admits a geometric decomposition. If the base and fibre are hyperbolic the only known examples are virtually products.

THEOREM 7. A closed 4-manifold $M$ is finitely covered by a manifold homotopy equivalent to one which fibres over an aspherical closed surface with fibre a hyperbolic surface if and only if $\pi_{1}(M)$ has a normal subgroup $G$ which is a $P D_{2}$-group with trivial centre and $\pi_{2}(M)=0$.

Proof. The conditions are clearly necessary. Conversely, if they hold then $\pi$ has one end. Hence $M$ is aspherical and $\pi=\pi_{1}(M)$ is a $P D_{4}$-group, since $\pi_{2}(M)=0$. Conjugation in $\pi$ determines an embedding of $\pi / G \cdot C_{\pi}(G)$ into $\operatorname{Out}(G)$, which is virtually of finite cohomological dimension. Since $G \cap C_{\pi}(G)=\zeta G=1$ the quotient $\pi / G$ is an extension of $\pi / G . C_{\pi}(G)$ by $C_{\pi}(G)$, and so v.c.d. $\pi / G \leq$ c.d. $C_{\pi}(G)+$ v.c.d. $\pi / G . C_{\pi}(G)<\infty$. Hence $\pi / G$ has a subgroup $\rho$ of finite index such that c.d. $\rho<\infty$. It now follows from Theorem 9.11 of $[\mathbf{B}]$ that $\rho$ is a $P D_{2}$-group. Therefore the cover of $M$ corresponding to the preimage of $\rho$ in $\pi$ is homotopy equivalent to the total space of an $F$-bundle over $B$, where $B$ and $F$ are aspherical surfaces with $\pi_{1}(B) \cong \rho$ and $\pi_{1}(F) \cong G$. // 
Note that $G . C_{\pi}(G) \cong G \times C_{\pi}(G)$ and so either $\pi$ is virtually a product or $C_{\pi}(G)$ is a free group. If $C_{\pi}(G) \cong Z$ then $G . C_{\pi}(G)$ is a $P D_{3}$-group and so $\pi / G . C_{\pi}(G)$ is virtually $Z$. Hence $\pi$ is virtually $H \times Z$, where $H$ is an extension of $Z$ by $G$.

Is a manifold satisfying the hypotheses of Theorem 7 homotopy equivalent to a quotient of a bundle space?

THEOREM 8. Let $B$ and $F$ be closed surfaces with $\chi(B)=0$ and $\chi(F)<0$. Let $E$ be the total space of the $F$-bundle over $B$ corresponding to a homomorphism $\theta: \pi_{1}(B) \rightarrow$ $\operatorname{Out}\left(\pi_{1}(F)\right)$. Then $E$ virtually has a geometric decomposition if and only if $\operatorname{Ker} \theta \neq 1$. Moreover

(i) $E$ admits the geometry $H^{2} \times E^{2}$ if and only if $\theta$ has finite image;

(ii) $E$ admits the geometry $H^{3} \times E^{1}$ if and only if $\operatorname{Ker} \theta \cong Z$ and $\operatorname{Im} \theta$ contains the class of a pseudo-Anasov diffeomorphism of $F$;

(iii) otherwise $E$ is not geometric.

Proof. Let $\pi=\pi_{1}(E)$. Since $E$ is aspherical, $\chi(E)=0$ and $\pi$ is not solvable the only possible geometries are $H^{2} \times E^{2}, H^{3} \times E^{1}$ and $\tilde{S L} \times E^{1}$. If $E$ has a proper geometric decomposition the pieces must all have $\chi=0$, and the only other geometry that may arise is $F^{4}$. In all cases the fundamental group of each piece has a nontrivial abelian normal subgroup.

If $\operatorname{Ker} \theta \neq 1$ then $E$ is virtually a cartesian product $N \times S^{1}$, where $N$ is the mapping torus of a self diffeomorphism $\psi$ of $F$ whose isotopy class in $\pi_{0}(\operatorname{Diff}(F)) \cong \operatorname{Out}\left(\pi_{1}(F)\right)$ generates a subgroup of finite index in $\operatorname{Im} \theta$. Since $N$ is a Haken 3-manifold it has a geometric decomposition and hence so does $E$. The mapping torus $N$ is an $H^{3}$ manifold if and only if $\psi$ is pseudo-Anasov. In that case the action of $\pi_{1}(N) \cong$ $\pi_{1}(F) \times_{\psi} Z$ on $H^{3}$ extends to an embedding $p: \pi / \sqrt{\pi} \rightarrow \operatorname{Isom}\left(H^{3}\right)$, by Mostow rigidity. Since $\sqrt{\pi} \neq 1$ we may also find a homomorphism $\lambda: \pi \rightarrow D<\operatorname{Isom}\left(E^{1}\right)$ such that $\lambda(\sqrt{\pi}) \cong Z$. Then $\operatorname{Ker} \lambda$ is an extension of $Z$ by $F$ and is commensurate with $\pi_{1}(N)$, so is the fundamental group of a Haken $H^{3}$-manifold, $\hat{N}$ say. Together these homomorphisms determine a free cocompact action of $\pi$ on $H^{3} \times E^{1}$. If $\lambda(\pi) \cong Z$ then $M=\pi \backslash\left(H^{3} \times E^{1}\right)$ is the mapping torus of a self homeomorphism of $\hat{N}$; otherwise it is the union of two twisted $I$-bundles over $\hat{N}$. In either case it follows from standard 3-manifold theory that since $E$ has a similar structure $E$ and $M$ are diffeomorphic.

If $\theta$ has finite image then we may construct an $H^{2} \times E^{2}$-manifold with group $\pi$ and which fibres over $B$ as in Theorem 5 above. Since such bundles are determined up to diffeomorphism by their fundamental groups $E$ admits this geometry.

Conversely, if a finite cover of $E$ has a geometric decomposition then we may assume that the cover is itself the total space of a surface bundle over the torus, and so we may assume that $E$ has a geometric decomposition and that $B \cong S^{1} \times S^{1}$. Let $\phi=\pi_{1}(F)$. Suppose first that $E$ has a proper geometric decomposition. Then $\pi=$ $\pi_{1}(E) \cong A *_{C} B$ or $A *_{C}$, where $C$ is solvable and of Hirsch length 3 , and where $A$ is the fundamental group of one of the pieces of $E$. Note that $\sqrt{A} \neq 1$. Let $\bar{A}=A / A \cap \phi$, $\bar{B}=B / B \cap \phi$ and $\bar{C}=C / C \cap \phi$. Then $\bar{\pi}=\pi / \phi \cong Z^{2}$ has a similar decomposition as 
$\bar{A} *_{\bar{C}} \bar{B}$ or $\bar{A} *_{\bar{C}}$. Now $C \cap \phi=1$ or $Z$, since $\chi(F)<0$. Hence $\bar{C} \cong Z^{2}$ and so $\bar{A}=$ $\bar{C}=\bar{B}$. In particular, $\operatorname{Im} \theta=\theta(A)$. But as $\sqrt{A} \cap \phi \leq \sqrt{\phi}=1$ and $\sqrt{A}$ and $A \cap \phi$ are normal subgroups of $A$ it follows that $\sqrt{A}$ and $A \cap \phi$ commute. Hence $\theta(A)$ is a quotient of $A / \sqrt{A} \cdot(A \cap \phi)$, which is abelian of rank at most 1 , and so $\operatorname{Ker} \theta \neq 1$.

If $E$ admits the geometry $H^{2} \times E^{2}$ then $\sqrt{\pi}=\pi \cap \operatorname{Rad}\left(\operatorname{Isom}\left(H^{2} \times E^{2}\right)\right)=\pi \cap$ $\left(\{1\} \times R^{2}\right) \cong Z^{2}$, by Proposition 8.27 of $[\mathbf{R g}]$. Hence $\theta$ has finite image.

If $E$ admits the geometry $H^{3} \times E^{1}$ then $\sqrt{\pi}=\pi \cap(\{1\} \times R) \cong Z$, by Proposition 8.27 of $[\mathbf{R g}]$. Hence $\operatorname{Ker} \theta \cong Z$ and $E$ is finitely covered a cartesian product $N \times S^{1}$, where $N$ is a hyperbolic 3-manifold which is also an $F$-bundle over $S^{1}$. The geometric monodromy of the latter bundle is a pseudo-Anasov diffeomorphism of $F$ whose isotopy class is in $\operatorname{Im} \theta$.

If $\rho$ is the group of an $\tilde{S L} \times E^{1}$-manifold then $\sqrt{\rho} \cong Z^{2}$ and $\sqrt{\rho} \cap K^{\prime} \neq 1$ for all subgroups $K$ of finite index, and so $E$ cannot admit this geometry. //

In particular, if $\chi(B)=0$ and $\theta$ is injective $E$ admits no geometric decomposition.

We shall assume henceforth that $B$ is also hyperbolic. Then $\chi(E)>0$ and $\pi_{1}(E)$ has no solvable subgroups of Hirsch length 3. Hence the only possible geometries on $E$ are $H^{2} \times H^{2}, H^{4}$ and $H^{2}(C)$. (These are the least well understood geometries, and little is known about the possible fundamental groups of the corresponding 4-manifolds).

THEOREM 9. Let $B$ and $F$ be closed hyperbolic surfaces, and let $E$ be the total space of the $F$-bundle over $B$ corresponding to a homomorphism $\theta: \pi_{1}(B) \rightarrow \operatorname{Out}\left(\pi_{1}(F)\right)$. Then the following are equivalent:

(i) $E$ admits the geometry $H^{2} \times H^{2}$;

(ii) $E$ is finitely covered by a cartesian product;

(iii) $\theta$ has finite image.

If $\operatorname{Ker} \theta \neq 1$ then $E$ does not admit either of the geometries $H^{4}$ or $H^{2}(C)$.

Proof. Let $\pi=\pi_{1}(E)$ and $\phi=\pi_{1}(F)$. If $E$ admits the geometry $H^{2} \times H^{2}$ it is virtually a cartesian product, by Theorem 5 , and so (i) implies (ii).

Suppose that $\pi$ has a subgroup $v$ of finite index which is a direct product $v=$ $\alpha . \beta \cong \alpha \times \beta$, where $\alpha$ and $\beta$ are normal subgroups of $v$ and $\alpha \cap \beta=1$. Let $G=\phi \cap \nu$. Then $\alpha, \beta$ and $G$ are each $P D_{2}$-groups with trivial centre, and $G$ is normal in $v$. Either $G \cap \alpha=1$ or $G \cap \beta=1$, for otherwise $Z^{2} \leq(G \cap \alpha)(G \cap \beta) \leq G$. Suppose that $G \cap \alpha=1$. Then $a g a^{-1} g^{-1}=1$ for all $a \in \alpha$ and $g \in G$, since $\alpha$ and $G$ are each normal in $v$. Hence $\alpha \leq C_{\pi}(G)$. An automorphism of $\phi$ which induces the identity on $G$ and on $\phi / G$ must be the identity, since $\zeta G=1$. Hence $C_{\pi}(\phi) \cap \alpha$ has finite index in $\alpha$. Since $\alpha \cap \phi=\alpha \cap G=1$ the projection of $\pi$ onto $\pi_{1}(B)=\pi / \phi$ maps $\alpha$ injectively, and since $\alpha$ and $\pi_{1}(B)$ are both $P D_{2}$-groups the image must have finite index. Therefore the image of $\theta$ is finite and so (ii) implies (iii).

If $\theta$ has finite image then $\operatorname{Ker} \theta \neq 1$ and $\pi / C_{\pi}(\phi)$ is a finite extension of $\phi$. Hence there is a homomorphism $p: \pi \rightarrow \operatorname{Isom}\left(H^{2}\right)$ with kernel $C_{\pi}(\phi)$ and with image a discrete cocompact subgroup. Let $q: \pi \rightarrow \pi_{1}(B)<\operatorname{Isom}\left(H^{2}\right)$. Then $(p, q)$ embeds $\pi$ as a 
discrete cocompact subgroup of Isom $\left(H^{2} \times H^{2}\right)$, and the closed 4-manifold $M=$ $\pi \backslash\left(H^{2} \times H^{2}\right)$ clearly fibres over $B$. Since such bundles are determined up to diffeomorphism by their fundamental groups $E$ admits the geometry $H^{2} \times H^{2}$. Hence (iii) implies (i).

If $\theta$ is not injective $Z^{2}<\pi$ and so $E$ cannot admit either of the geometries $H^{4}$ or $H^{2}(C)$, by Theorem 9 of $[\operatorname{Pr43}]$. //

In particular, if $\chi(B) \neq 0$ and $\theta$ is not injective but has infinite image then $E$ is not geometric. Can such a bundle space have a geometric decomposition into pieces of type $H^{2} \times H^{2}$ ? The equivalence of (ii) and (iii) is Lemma 4 of [Jo94].

Are there any such bundle spaces $E$ which admit one of the geometries $H^{4}$ or $H^{2}(C)$ ? Orientable manifolds with geometry $H^{4}$ have signature 0 , whereas if $M$ is a $H^{2}(C)$-manifold $\chi(M)=3 \sigma(M)>0$. It is shown in [Me73] that if $B$ and $F$ are orientable $\sigma(E)=-\theta^{*} \tau \cap[B]$, where $\tau$ is a class in $H^{2}\left(\operatorname{Out}\left(\pi_{1}(F)\right) ; Z\right)$ induced from an explicitly described class in $H^{2}\left(S p_{2 g}(Z) ; Z\right)$ via the natural representation of $\operatorname{Out}\left(\pi_{1}(F)\right)$ as symplectic isometries of the intersection form on $H_{1}(F ; Z) \cong Z^{2 g}$. In particular, if $F$ has genus $g=2$ then $\sigma(E)=0$ and so $E$ does not admit the geometry $H^{2}(C)$.

Every closed orientable $H^{2} \times H^{2}$ - or $H^{2}(C)$-manifold has a 2-fold cover which is a complex analytic surface, whereas no closed $H^{4}$-manifold admits a complex structure [W186]. If $E$ is a complex surface it can be shown that $B$ is orientable and $p$ is homotopic to a holomorphic map, for some complex structure on $B$. Must $p$ be isotopic to a holomorphic submersion? By a theorem of Parshin, for each $n>0$ there are (up to deformation) only finitely many complex analytic surfaces $S$ with $\chi(S)=n$ which admit holomorphic submersions onto a nonsingular complex curve. (See [Jo94]). If $E$ is a complex analytic surface and $h: E \rightarrow B$ is a holomorphic fibre bundle then $E$ is finitely covered by a cartesian product of complex curves. (See $\S \mathrm{V} .6$ of [BPV]). However there are such holomorphic submersions in which $\sigma(E) \neq 0$ and so which are not virtually products. (See $\S \mathrm{V} .14$ of [BPV]). The known examples are not quotients of the unit ball in $C^{2}$ and so admit no geometry. Any holomorphic submersion with base of genus at most 1 or fibre of genus at most 2 is a holomorphic fibre bundle [Ka68].

The final theorem strengthens a remark made in $\S \mathrm{VI} .7$ of $[\mathbf{H}]$.

THEOREM 10. A closed 4-manifold $M$ with fundamental group $\pi$ is s-cobordant to an $X$-manifold where $X=H^{2} \times H^{2}, H^{4}$ or $H^{2}(C)$ if and only if $\pi$ is torsion free and is isomorphic to a discrete uniform subgroup of $\operatorname{Isom}(X)$ and $\chi(M)=\chi(\pi \backslash X)$.

Proof. The conditions are clearly necessary. Suppose that they hold. Then $\chi(M)>0$ and $\pi$ is infinite and residually finite. Hence there is a subgroup $\sigma$ of finite index such that the associated covering spaces $M_{\sigma}$ and $\sigma \backslash X$ are orientable and $\chi(M)=\chi(\sigma \backslash X)>2$. In particular, $H^{2}(M ; Z)$ has elements of infinite order. Since the classifying map $c_{M_{\sigma}}: M_{\sigma} \rightarrow \sigma \backslash X$ is 2-connected it induces an isomorphism on $H^{2}$ and hence is a degree-1 map, by Poincaré duality. Therefore it is a homotopy equivalence, by Theorem II.3 of $[\mathbf{H}]$, and so $M$ is aspherical. Hence $c_{M}: M \rightarrow \pi \backslash X$ is a homotopy equivalence. 
It now follows from the work of Farrell and Jones [FJ90] that $\mathrm{Wh}(\pi)=$ $\mathrm{Wh}(\pi \times Z)=0$ and that $M \times S^{1}$ is homeomorphic to $(\pi \backslash X) \times S^{1}$. Hence $M$ and $\pi \backslash X$ are $s$-cobordant. //

ACKNOWLEDGMENT. I would like to thank M.Farber for asking the question that prompted this work, and J. S. Crisp for finding the examples of Seifert fibred 3manifolds used in the discussion of the geometry $S^{3} \times E^{1}$ in $\$ 2$.

\section{References}

[BPV] Barth, W., Peters, C. and Van de Ven, A., Compact Complex Surfaces, Ergebnisse der Mathematik und ihrer Grenzgebiete 3 Folge, Bd 4, Springer-Verlag, Berlin - Heidelberg - New York Tokyo (1984).

[B] Bieri, R., Homological Dimension of Discrete Groups, Queen Mary College Mathematics Notes, London (1976).

[CH96] Cobb, R. and Hillman, J. A., $S^{2}$-bundles over aspherical surfaces and 4-dimensional geometries, Proc. Amer. Math. Soc. (in press).

[CR72] Conner, P. E. and Raymond, F., Holomorphic Seifert fiberings, in Proceedings of the Second Conference on Compact Transformation Groups II, Lecture Notes in Mathematics 299 (1972), 124204.

[Eb80] Eberlein, P., Lattices in spaces of nonpositive curvature, Ann. Math. 111 (1980), 435-476.

[Ep87] Epstein, D. B. A., Complex hyperbolic geometry, in Analytical and Geometrical Aspects of Hyperbolic Space, London Mathematical Society Lecture Notes Series 111, Cambridge University Press, Cambridge (1987), 93-111.

[Fa74] Farrell, F. T., The second cohomology group of $G$ with coefficients $Z / 2 Z[G]$, Topology 13 (1974), 313-326.

[Fa75] Farrell, F. T., Poincaré duality and groups of type FP, Comment. Math. Helvetici 50 (1975), 187195.

[FJ90] Farrell, F. T. and Jones, L. E., Rigidity and other topological aspects of compact nonpositively curved manifolds, Bull. Amer. Math. Soc. 22 (1990), 59-64.

[F] Filipkiewicz, R. O., Four-Dimensional Geometries, PhD thesis, University of Warwick (1984).

[H] Hillman, J. A., The Algebraic Characterization of Geometric 4-Manifolds, London Mathematical Society Lecture Notes Series 198, Cambridge University Press (1994).

[Hi95] Hillman, J. A., Flat 4-manifold groups, New Zealand Math. J. 24 (1995), 29-40.

[Jo94] Johnson, F. E. A., A group theoretic analogue of the Parshin-Arakelov rigidity theorem, Archiv der Math. 63 (1994), 354-361.

[Ka68] Kas, A., On deformations of a certain type of irregular algebraic surface, American J. Math. 90 (1968), 789-804.

[Ke83] Kerckhoff, S. P., The Nielsen realization problem, Ann. Math. 117 (1983), 235-265.

[Kr 90] Kropholler, P. H., An analogue of the torus decomposition theorem for certain Poincare duality groups, Proc. London Math. Soc. 60 (1990), 503-529.

[M] Margulis, G. A., Discrete Subgroups of Lie Groups, Ergebnisse der Mathematik und ihrer Grenzgebiete 3 Folge Bd. 17, Springer-Verlag, Berlin - Heidelberg - New York (1991).

[Me73] Meyer, W., Die Signatur von Flächenbundeln, Math. Ann. 201 (1973), 239-264.

[NS85] Nicas, A. and Stark, C. W., $K$-Theory and surgery of codimension-two torus actions on aspherical manifolds, J. London Math. Soc. 31 (1985), 175-185.

[Ni95] Nimershiem, B. E., All flat 3-manifolds appear as cusps of hyperbolic four-manifolds, preprint, Franklin and Marshall College (1995).

[Oh90] Ohba, K., On fiber bundles over $S^{1}$ having small Seifert manifolds as fibers, J. Fac. Sci. Tokyo 37 (1990), 659-702.

[Pr43] Preissmann, A., Quelques propriétés globales des espaces de Riemann, Comment. Math. Helvetici 15 (1943), 175-216.

[Rg] Raghunathan, M. S., Discrete Subgroups of Lie Groups, Ergebnisse der Mathematik 68, SpringerVerlag, Berlin - Heidelberg - New York (1972).

[Sc92] Scott, P., The characteristic submanifold in higher dimensions, preliminary report, Abstracts AMS *876-57-22 (1992). 
[Sh63] Shimizu, H., On discontinuous groups operating on the product of upper half planes, Ann. Math. 77 (1963), 33-71.

[St84] Stark, C. W., Structure sets vanish for certain bundles over Seifert manifolds, Trans. Amer. Math. Soc. 285 (1984), 603-615.

[Th82] Thurston, W. P., Three dimensional manifolds, Kleinian groups and hyperbolic geometry, Bull. Amer. Math. Soc. (N.S.) 6 (1982), 357-381.

[Ue90] Ue, M., Geometric 4-manifolds in the sense of Thurston and Seifert 4-manifolds I, J. Math. Soc. Japan 42 (1990), 511-540.

[Ue91] Ue, M., Geometric 4-manifolds in the sense of Thurston and Seifert 4-manifolds II, J. Math. Soc. Japan 43 (1991), 149-183.

[W185] Wall, C. T. C., Geometries and geometric structures in real dimension 4 and complex dimension 2, in Geometry and Topology (edited by J. Alexander and J. Harer), Lecture Notes in Mathematics 1167, Springer-Verlag, Berlin - Heidelberg - New York (1985), 268-292.

[W186] Wall, C. T. C., Geometric structures on complex analytic surfaces, Topology 25 (1986), 119-153.

[Zi85] Zimmermann, B., Zur klassification höherdimensionaler Seifertsche faserräume, in Low Dimensional Topology (edited by R. Fenn), London Mathematical Society Lecture Notes Series 95, Cambridge University Press (1985).

\section{Jonathan A. Hillman}

School of Mathematics and Statistics, The University of Sydney, Sydney, NSW 2006,

Australia 